\title{
Face Recognition Using Topological Manifolds Learning
}

\author{
Cao Wenming and Lu Fei \\ Institute of Intelligent Information System, Information College, \\ Zhejiang University of Technology, \\ Hangzhou 310032, China \\ luf@zjut.edu.cn
}

\begin{abstract}
An algorithm of PCA face recognition based on topological manifolds theory is proposed, which based on the sample sets' topological character in the feature space which is different from "classification". Compare with the traditional PCA+ NN algorithm, experiments prove its efficiency.
\end{abstract}

\section{Introduction}

Automatic human face recognition is attractive in personal identification, which widely used in security, bank and commerce department. Compared with other biometric identification techniques such as fingerprint recognition and retina recognition, it is directly, friendly and convenient. So the research of face recognition is become very hot ${ }^{[1]}$. Face recognition Based on geometrical features is proposed by D.L.Domoho ${ }^{[2]}$, there are other algorithm such as based on deformable template, the character of eyes, neural networks ${ }^{[5][6][7]}$ and the generalized symmetry transform ${ }^{[3]}$, In this paper we introduced an novel face recognition algorithm based on topological manifolds theory ${ }^{[4]}$, experiments prove its efficiency.

\section{Principal Component Analysis(PCA)}

At common ,a face image is described a high dimensional vector in image space. Facial image can be regarded as a point in the space. Fig 1. shows this idea graphically. Because there are strong symmetry structure of face(eyes, nose, and mouth and so on),so usually the vectors is relative. We can find that images of a people congregate in a certain area in space. So we can project face images into an array of eigenvector, which we obtain from covariance matrix of the trained face images.

Suppose the face image to be size of L by L, this image can be described as a L2 high dimensional vector or a point in a space of L2 high dimension. A set of image correspond to a set of point. Because the distribution is not at random, so we can project it to a low dimensional subspace. PCA is a usual dimension reduction method which gives the basis vector for this subspace. Each basis vector is eigenvector of the covariance matrix corresponding to the original face images. 


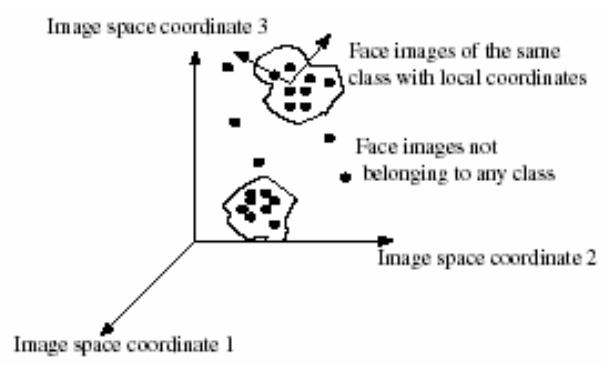

Fig. 1. Image space

Supposed $I_{1}, \quad I_{2} \ldots \ldots, \quad I s$ as a training set, its' average face can be defined by:

$$
A=\frac{1}{S} \sum_{i=1}^{S} I i
$$

The margin of a face image and average face is $Y_{i}=I_{i}-A$.Covariance matrix $\mathrm{C}$ is defined by:

$$
C=\frac{1}{S} \sum_{i=1}^{S} Y_{i} \cdot Y_{i}^{T}
$$

Select the maximum $\mathrm{M}$ eigenvector, and then get corresponding value of every image:

$$
W_{i K}=E_{K}^{T} \cdot\left(I_{i}-A\right) \quad \forall i, K
$$

$E_{K}^{T}$ is the maximum M eigenvector, the range of $K$ is from 1 to $\mathrm{M}$.

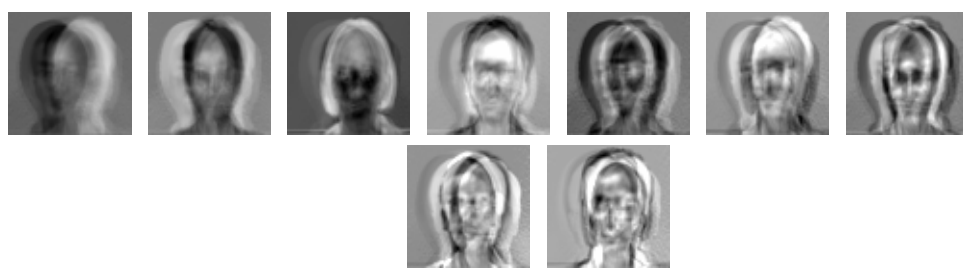

Fig. 2. illuminates face reconstruction corresponding to the maximum 10 eigenvector in experiments

Test image $I_{\text {test }}$ project to face space as the algorithm :

$$
W_{\text {testK }}=E_{K}^{T} \cdot\left(I_{\text {test }}-A\right) \quad \forall K .
$$




\section{Algorithm of PCA Face Recognition Based on Topological Manifolds Learning}

1) Reduce image space dimension via PCA, project the training image into a low dimensional space, and get the new image vector of low dimension.

2) Form Topological Manifolds in low dimensions space. The method of form Topological Manifolds is:

Consider the training sample set is $S$, the size of this set is $N$,let $\mathrm{i}=1$.

Step 1:Select an image $a$ in set $S$ at random,

Step 2: Calculate distance between $a$ and the remain training set $S-\{a\}$. Get sample $b$ which is nearest to $a$.

Step 3: Form Topological Manifolds $\mathrm{A}[\mathrm{i}$ ] used sample $a$ and $b$, let $\mathrm{i}=\mathrm{i}+1$

Step 4: Abandon image $a$ from set $S$, then $S=S-\{a\}, N=N-1$, let $a=b$

step 5: if $N=1$, the process is complete, otherwise repeat from step1 to step 5 .

Fig 3 shows the result of different people's Topological Manifolds

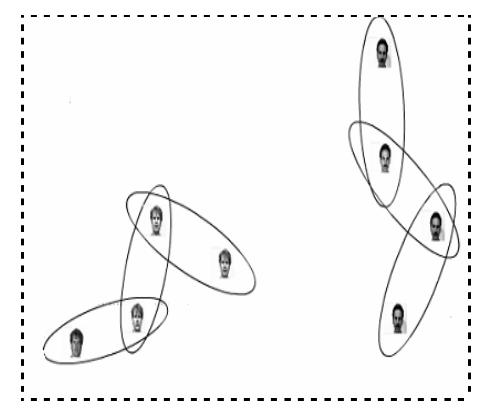

Fig. 3. Different people's on Topological Manifolds

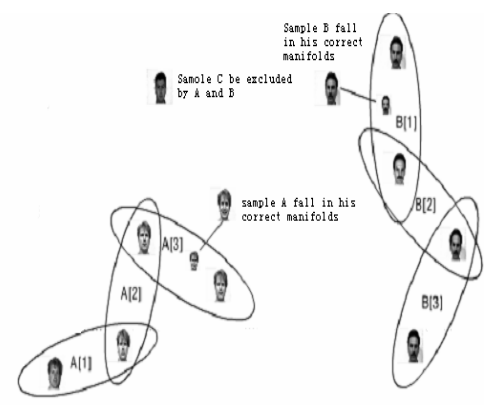

Fig. 4. The recognition process based Topological Manifolds

3) Project every test sample $T S_{i}$ in test set $T_{-} S$ to image space of the same low dimension, and then calculate the distance between the new image vector and every people Topological Manifolds. If it lies in the a people's Topological Manifolds, we can confirm it belong to this people. If the test image vector does not lie in any people's Topological Manifolds, we can say we do not recognize this sample (Fig 4 demonstrates this process graphically.).

\section{Experiment Result}

In experiments we used both UMIST and Yale database. The image of Yale database have different illumination and expression, so we use it to analyse the affection of illumination and expression. Meanwhile we use UMIST database to analyse the 
affection of face position. We compare the traditional PCA $+\mathrm{NN}$ algorithm with PCA+ Topological manifolds theory.

Experiment 1: In Yale database we select 8 people, and select 6 images of every of the 8 people to train the neural network. And we test the remain 5 images and the other 7people's images.

Table 1. Comparison of the efficiency used Yale database

\begin{tabular}{|c|c|c|c|}
\hline & Correct rate & Rejection rate & Error rate \\
\hline PCA+ NN & $70 \%$ & 0 & $30 \%$ \\
\hline $\begin{array}{c}\text { PCA+Topological } \\
\text { Manifolds }\end{array}$ & $95 \%$ & $100 \%$ & $5 \%$ \\
\hline
\end{tabular}

Under the variation of illumination and expression, we can see from above table, the correct rate of PCA+Topological manifolds is higher than that of PCA $+\mathrm{NN}$. Also it can correctly confirm all of the unacquainted image. So we can say that, under the variation of illumination and expression, PCA+Topological manifolds is superior to $\mathrm{PCA}+\mathrm{NN}$.

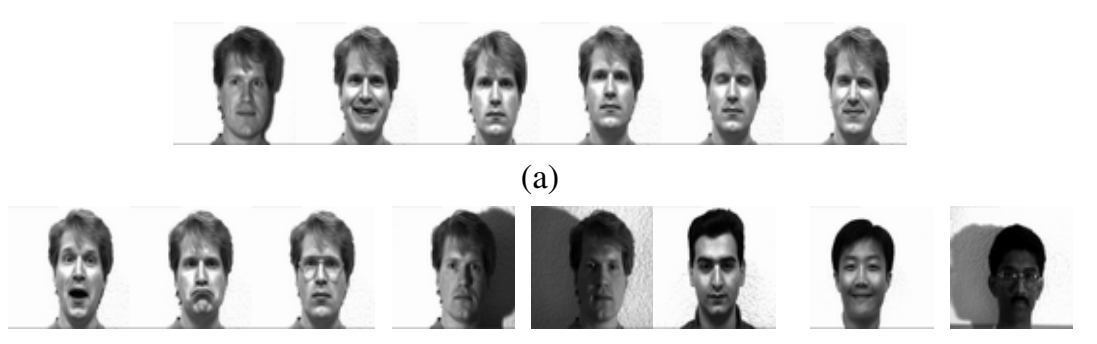

(b)

Fig. 5. The training image (a) and testing image(b) in Yale database

Experiment 2: In UMIST database we select 15 people, and select 8 images of every of the 15 people to train the neural network. And we test the remain 2 images and the other 5 people's images.

Table 2. Comparison of the efficiency used UMIST database

\begin{tabular}{|l|c|c|c|}
\hline & Correct rate & Rejection rate & Error rate \\
\hline PCA+ NN & $72.5 \%$ & 0 & $27.5 \%$ \\
\hline PCA+Topological Manifolds & $85 \%$ & $100 \%$ & $15 \%$ \\
\hline
\end{tabular}


Under the variation of face position, we can see from above table, the correct rate of PCA+Topological manifolds is higher than that of PCA+ NN. Also it can correctly confirm all of the unacquainted image. So we can say that, under the variation of illumination and expression, PCA+Topological manifolds is superior to PCA+ NN.

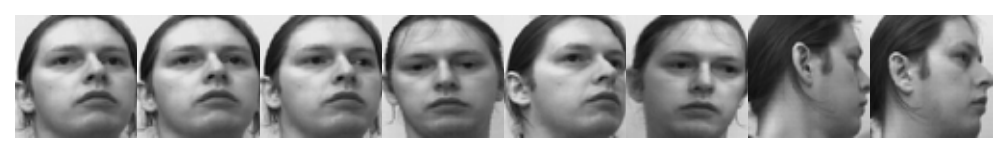

(a)

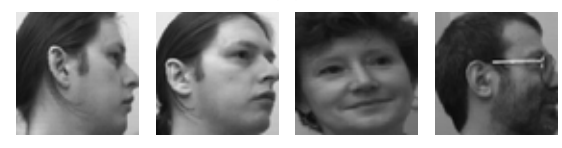

(b)

Fig . 6. The training image (a) and testing image(b) in UMIST database

Experiment 3: We test the efficiency between the traditional PCA+ NN algorithm and PCA+ Topological manifolds theory when the dimension of eigenvector changed. If heighten the size of eigenvector, recognition rate increased, however complexity increased as well. when $\mathrm{M}>30$, the recognition rate do not increase obviously. Because an algorithm of PCA face recognition based on topological manifolds theory is based on the sample sets' topological character in the feature space which is different from "classification", its recognition rate is much better.

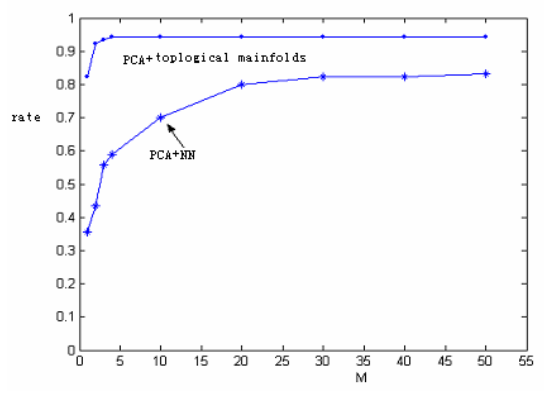

Fig. 7. Comparison of the efficiency when $M$ is changed

\section{Conclusion and Problem}

In the experiment we can draw conclusion below

1) Face recognition based on topological manifolds do not recognize every people not trained, which is better closer to the function of human being. 
2) When add the new trained sample ,topological manifolds structure of original samples do not change.

3) In our experiments, we must make sure of the preprocess is continuous project, otherwise it will affect the efficiency.

Meanwhile there are some problem existed. When forming Topological Manifolds, the size of topological manifolds is important. If the size is bigger ,the chance of a image to fall in the topological manifolds increased, which will bring increase of correct recognition rate, as well as error recognition rate.

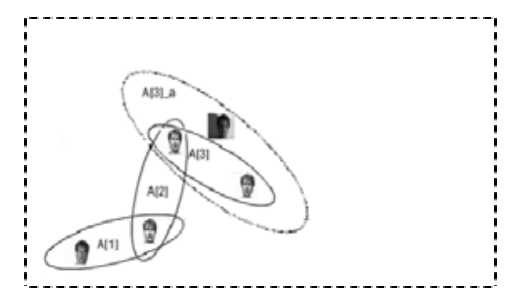

Fig. 8. The structure of different size

The number of a people's sample affect the structure of a people's topological manifolds. When the number of sample is increasing, the chance of the image to fall in it is increasing also., so the correct recognition rate is higher.

\section{References}

1. 1.Deslauiers,G.andDubuc,s. Symmetric iterative interpolation processes. Constr. Approx., (1989) 5(1):49-682

2. Monterey,Californla, Wavelet mathe -matics and application CRC Press, Inc, 1994

3. Ami Hartegen. Multi-resolution Represe -ntation of data: Ageneral Frame works, SIAM. J. numer. Anal (1996) 33(3) $1205-1255$

4. Wang ShouJue, A new development on ANN in China - Biomimetic pattern recognition and multi weight vector neurons, LECTURE NOTES IN ARTIFICIAL INTELLIGENCE 2639: 35-43, 2003

5. Wang shoujue, Xu jian, Wang xianbao, Qin hong, Multi Camer Human Face Personal Identification System based on the biomimetic pattern recognition, ACTA ELECTRONICA SINICA, Vol.31 No.1,Jan. 2003 1-3

6. Wenming Cao, Feng Hao, Shoujue Wang: The application of DBF neural networks for object recognition. Inf. Sci. (2004) 160(1-4): 153-160

7. Wenming Cao, Jianhui Hu, Gang Xiao, Shoujue Wang. Iris Recognition Algorithm Based on Point Covering of High-Dimensional Space and Neural Network, LECTURE NOTES IN ARTIFICIAL INTELLIGENCE 3587, pp. 305 - 313, 2005. 